This item was submitted to Loughborough's Research Repository by the author.

Items in Figshare are protected by copyright, with all rights reserved, unless otherwise indicated.

\title{
Liquid film coating a fiber as a model system for the formation of bound states in active dispersive-dissipative nonlinear media
}

PLEASE CITE THE PUBLISHED VERSION

http://dx.doi.org/10.1103/PhysRevLett.103.234501

PUBLISHER

(C) American Physical Society

VERSION

VoR (Version of Record)

LICENCE

CC BY-NC-ND 4.0

\section{REPOSITORY RECORD}

Duprat, Camille, Frédérique Giorgiutti-Dauphiné, Dmitri Tseluiko, Sergey Saprykin, and Serafim Kalliadasis. 2019. "Liquid Film Coating a Fiber as a Model System for the Formation of Bound States in Active Dispersivedissipative Nonlinear Media”. figshare. https://hdl.handle.net/2134/15488. 
This item was submitted to Loughborough's Institutional Repository (https://dspace.lboro.ac.uk/) by the author and is made available under the following Creative Commons Licence conditions.

\section{creative
commons}

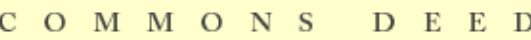

Attribution-NonCommercial-NoDerivs 2.5

You are free:

- to copy, distribute, display, and perform the work

Under the following conditions:

Attribution. You must attribute the work in the manner specified b the author or licensor.

Noncommercial. You may not use this work for commercial purposes.

No Derivative Works. You may not alter, transform, or build upon this work.

- For any reuse or distribution, you must make clear to others the license terms of this work.

- Any of these conditions can be waived if you get permission from the copyright holder.

Your fair use and other rights are in no way affected by the above.

This is a human-readable summary of the Leqal Code (the full license).

\section{Disclaimer 만}

For the full text of this licence, please go to: http://creativecommons.org/licenses/by-nc-nd/2.5/ 


\title{
Liquid Film Coating a Fiber as a Model System for the Formation of Bound States in Active Dispersive-Dissipative Nonlinear Media
}

\author{
C. Duprat, ${ }^{1}$ F. Giorgiutti-Dauphiné, ${ }^{1}$ D. Tseluiko, ${ }^{2}$ S. Saprykin, ${ }^{2}$ and S. Kalliadasis ${ }^{2}$ \\ ${ }^{1}$ CNRS, Université Pierre et Marie Curie, Université Paris-Sud, Lab FAST, Bât 502, Campus Universitaire, Orsay 91405, France \\ ${ }^{2}$ Department of Chemical Engineering, Imperial College London, London SW7 2AZ, United Kingdom
}

(Received 3 July 2009; published 2 December 2009)

\begin{abstract}
We analyze the coherent-structure interaction and the formation of bound states in active dispersivedissipative nonlinear media using a viscous film coating a vertical fiber as a prototype. The coherent structures in this case are droplike pulses that dominate the evolution of the film. We study experimentally the interaction dynamics and show evidence for formation of bound states. A theoretical explanation is provided through a coherent-structures theory of a simple model for the flow.
\end{abstract}

PACS numbers: 47.20.Ma, 47.35.Fg, 47.54. $-\mathrm{r}$, 68.15.+e

Bound states, i.e., composites of two or more building blocks behaving as single objects, appear in a wide variety of physical settings, from atomic physics and quantum mechanics to biological physics and complex fluids [1]. In this Letter, we demonstrate experimentally bound-stateformation phenomena in low-Reynolds-number interfacial hydrodynamics. Our experimental system consists of a viscous film flowing down a vertical fiber. The film is always unstable and spontaneously breaks up into a droplike wave train [2]. Streamwise viscous diffusion plays a dispersive role that dramatically affects the wave selection, speeds, and shapes [3]. This system is a simple example of a nonlinear medium with energy supply, energy dissipation, and dispersion, and as such it can be used as a prototype for the study of generic features of the patternformation dynamics of open-flow hydrodynamic and other systems. For example, the instability can be either absolute or convective [3]. In the latter case, the interface is dominated by droplike interacting pulses. We demonstrate that for sufficiently strong dispersion, such pulses can form bound states.

A qualitative theoretical explanation of the observed bound-state-formation phenomena is provided with a coherent-structures theory for the generalized KuramotoSivashinsky (gKS) equation. This equation has been postulated in the literature as a prototype for the study of patternformation dynamics and spatiotemporal complexity in active dispersive-dissipative media. For sufficiently small dispersion, it exhibits spatiotemporal chaos while sufficiently large dispersion "regularizes" the solution in favor of a train of solitary pulses which continuously interact with each other [4]. Such "dissipative solitons," as they are referred to by Christov and Velarde [4], are due to a precise balance between nonlinearity, energy supply at long wavelengths, and energy dissipation at short ones, and they appear in many different contexts [5]. Coherent-structures theories have been formulated based on the idea of weak interaction between neighboring structures, e.g., Ref. [6]. For the gKS equation, previous efforts [7] appear to have overlooked important details, in particular, in relation to the spectra of the operators of the linearized system around the pulses and their adjoints. We offer for the first time a rigorous analysis of the spectra of these operators and provide precise criteria for the existence of bound states of pulses of the gKS equation.

A sketch of the experimental setup is shown in Fig. 1(a). Rhodorsil silicon oil v50 (density $\rho=963 \mathrm{~kg} / \mathrm{m}^{3}$, dynamic viscosity $\mu=48 \times 10^{-3}$ Pas, surface tension $\gamma=$ $20.8 \times 10^{-3} \mathrm{~N} / \mathrm{m}$, and capillary length $\kappa^{-1}=1.5 \mathrm{~mm}$ at $25^{\circ} \mathrm{C}$ ) flows on vertical nylon fibers of $1.5 \mathrm{~m}$ in length. Notations are shown in Fig. 1(b). $x$ denotes the streamwise direction along the fiber. To ensure strong curvature effects, radii $R$ are chosen to be small compared to the capillary length, $0.2 \mathrm{~mm}<R<0.475 \mathrm{~mm}$, i.e., $R / \kappa^{-1}<$ 0.32 . A balance between viscous drag and gravity gives a characteristic speed $U_{0}=\rho g h_{0}^{2} / 2 \mu$. The relevant dimensionless groups are the Reynolds number $\operatorname{Re}=\rho U_{0} h_{0} / \mu$ and the Weber number $\mathrm{We}=\gamma / \rho U_{0}^{2} h_{0}$. Parameters are chosen so that both surface tension and viscosity effects are
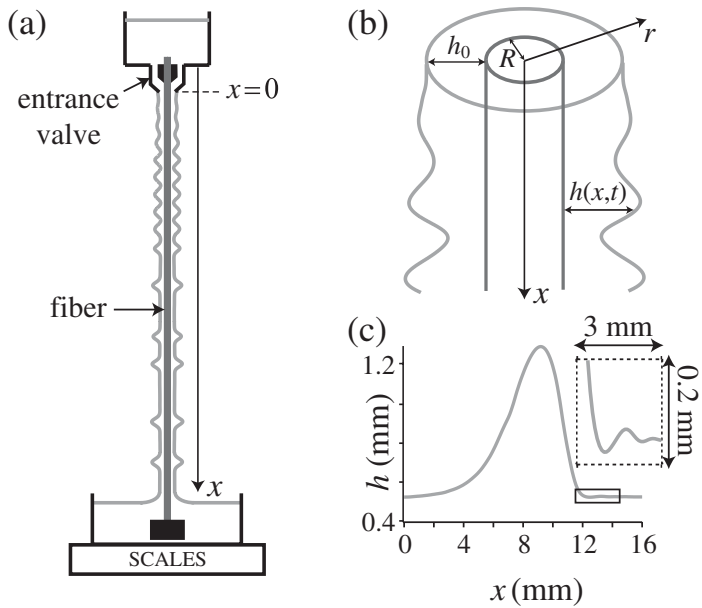

FIG. 1. (a) Experimental setup, (b) notations, and (c) typical solitary-pulse shape $(R=0.45 \mathrm{~mm})$. 
important, i.e., $0.6 \mathrm{~mm}<h_{0}<0.95 \mathrm{~mm}$ giving $3.5 \mathrm{~cm} / \mathrm{s}<$ $U_{0}<8.8 \mathrm{~cm} / \mathrm{s}, 30>\mathrm{We}>3$, and $0.4<\operatorname{Re}<1.7$.

The initially flat film breaks up into a regular droplike train of coherent structures, each of which resembles the pulse shown in Fig. 1(c). This instability results primarily from the capillary pressure induced by the azimuthal curvature (Rayleigh-Plateau instability); the pressure induced by the axial curvature has a stabilizing effect. Here we focus on the regime where the Rayleigh-Plateau instability competes with the viscous dispersive effect.

Spatiotemporal diagrams are obtained with a linear camera. The noise-driven dynamics is depicted in Fig. 2. The primary quasiregular wave train is destabilized by numerous coalescence and capillary drainage enhanced events leading to the formation of saturated pulses [see Fig. 2(a)]. After a certain distance, typically $80 \mathrm{~cm}$, no more coalescence occurs and the saturated pulses rearrange themselves through attractions and repulsions [see $\mathbf{A}$ and $\mathbf{B}$, respectively, in Fig. 2(b)]. Visualization of these events is obtained with a fast digital camera [see Figs. 2(c) and 2(d), respectively]. During attraction (repulsion) the upstream pulse $\mathbf{1}$ accelerates (decelerates) while the downstream pulse $\mathbf{2}$ decelerates (accelerates). The pulses then lock on at a given distance, which remains constant, and both travel at constant speed forming a bound state. Although no obvious regular pattern is observed down the fiber, statistics on the separation distances between successive pulses performed at the bottom of the fiber (at around $x=1.4 \mathrm{~m}$ ) and with a large ensemble averaging reveal a clear reorganization of the system with certain distances being selected. Indeed, in the histogram presented in Fig. 2(b), we observe that, although the distribution of distances is broad, four specific distances stand out: $d_{1} \approx 1.2 \mathrm{~cm}, d_{2} \approx 1.8 \mathrm{~cm}, d_{3} \approx 2.6 \mathrm{~cm}$, and $d_{4} \approx 3.35 \mathrm{~cm}$. The system appears to be heading towards organization. We have explored the parameter space by changing the fiber radius and the initial film thickness. Bound states are always observed with two to four preferred distances. Pulse rearrangement seems to be mainly promoted by interaction without substantial mass exchange. Waves then only interact through their tails whose specific structure is responsible for the observed phenomena. The characteristic profile of the waves is shown in Fig. 1(c). Surface tension and inertia lead to the front capillary ripples while viscous friction suppresses them. The steepening of the front is a nonlinear kinematic effect while inertia, gravity, and viscous drag are responsible for the gentle monotonically increasing back edge. As two nearly identical pulses approach each other, the back slope of the downstream pulse $\mathbf{2}$ overlaps with a minimum (maximum) of one of the periodic capillary waves preceding the upstream pulse 1. This generates a negative (positive) differential capillary pressure in the liquid $\left(\Delta p \sim-\gamma h_{x x}\right)$ that drains fluid from $\mathbf{2}$ to $\mathbf{1}$ (1 to 2$)$. This depresses (elevates) the back slope of the front pulse $\mathbf{2}$ and increases (decreases) its curvature. The generated differential capillary pressure then drains a small amount of fluid out of (into) the crest of $\mathbf{2}$, slightly changing its amplitude and its speed, while the back pulse $\mathbf{1}$ experiences an opposite change in amplitude. The front pulse then moves slightly slower (faster) than the back pulse, and the pulses attract (repel) each other [see Figs. 2(c) and 2(d)]. This mechanism implies not only the existence of several possible distances between pulses but also that the distances
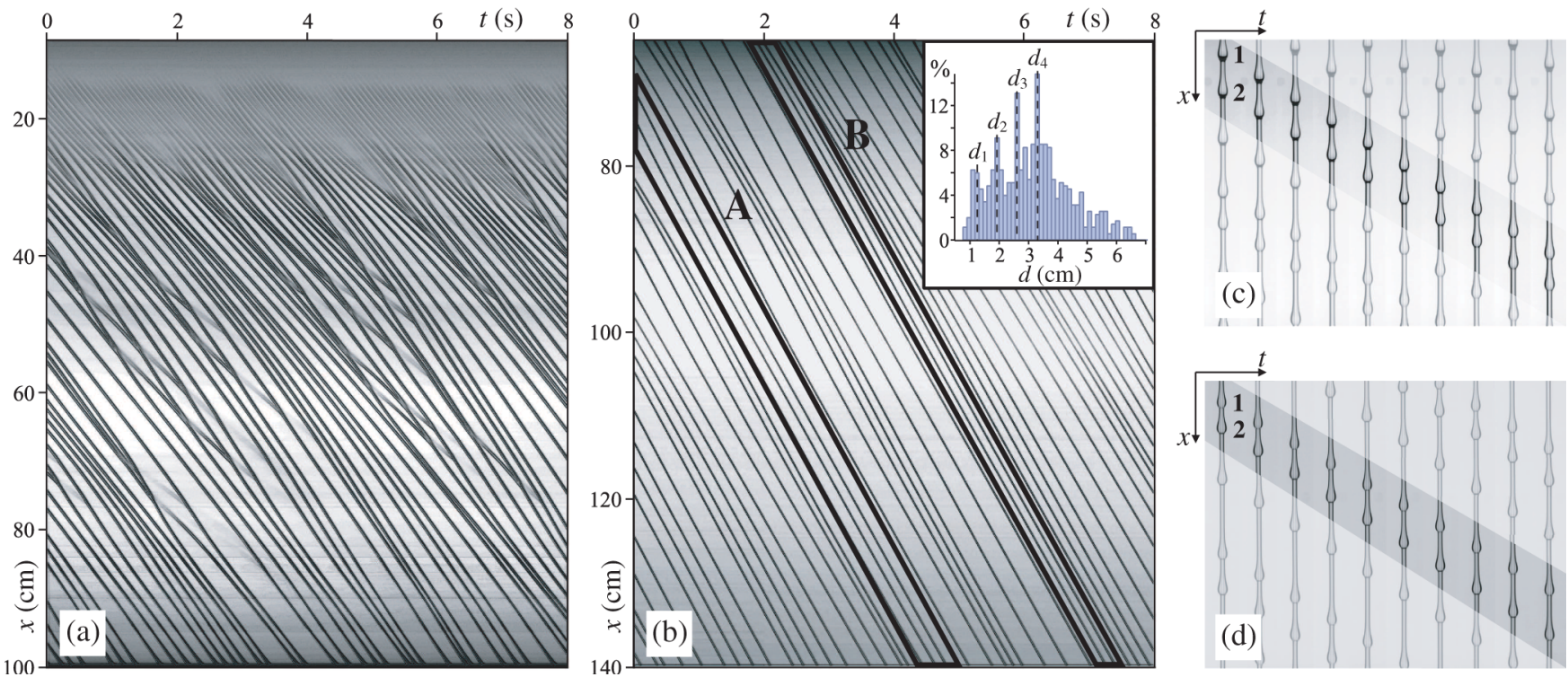

FIG. 2 (color online). Experimentally obtained world lines tracking the wave peaks during noise-driven evolution for $R=0.45 \mathrm{~mm}$, $\mathrm{We}=18, \operatorname{Re}=0.56$, and $\delta=0.4$ : (a) $0.07 \mathrm{~m}<x<1 \mathrm{~m}$ and (b) $0.65 \mathrm{~m}<x<1.4 \mathrm{~m}$; inset: histogram of the distances between pulses at the bottom of the fiber, $1.2 \mathrm{~m}<x<1.4 \mathrm{~m}$. (c) and (d) show consecutive images (height: $9 \mathrm{~cm}$ ) demonstrating attraction $(\mathrm{We}=5, \operatorname{Re}=1.2$, and $\delta=0.39)$ and repulsion $(\mathrm{We}=3.4, \mathrm{Re}=1.5$, and $\delta=0.42)$, respectively, for $R=0.35 \mathrm{~mm}$. Time between two consecutive images is $0.08 \mathrm{~s}$. 
between the neighboring prominent peaks in the histogram of Fig. 2(b) are roughly the same, i.e., $d_{2}-d_{1} \approx d_{3}-$ $d_{2} \approx d_{4}-d_{3}$, and are related to the period of the oscillations of the front tail of the pulse. An explanation for this effect is given below.

We start by deriving a simple model for the flow. The governing equations are the Navier-Stokes in cylindrical coordinates with the wall and the free-surface boundary conditions. We assume $R / h_{0}=O\left(\epsilon^{-1}\right)$, We $=O\left(\epsilon^{-2}\right)$, and $\operatorname{Re}=O(\epsilon)$, where $\epsilon \ll 1$ is the long-wave or film parameter, e.g., Ref. [8]. Through a weakly nonlinear expansion in which the amplitude deviation from $h_{0}$ is taken of $O\left(\epsilon^{2}\right)$, we obtain the following equation:

$$
H_{T}-c_{\delta} H_{X}+H H_{X}+H_{X X}+\delta H_{X X X}+H_{X X X X}=0,
$$

already in a frame moving with the velocity of a pulse. $X$, $T$, and $H$ are defined through

$$
\begin{aligned}
x & =h_{0} A^{-1 / 2}\left(X+c_{\delta} T\right)+3 B\left[2+\frac{2}{3} \alpha-\frac{1}{2} \alpha^{2}\right] T, \\
t & =3 U_{0}^{-1} B T, \quad H=12 A^{1 / 2} B h_{0}^{-2}\left(h-h_{0}\right),
\end{aligned}
$$

where $A=8 / 5 \mathrm{We}+\alpha^{2}, \quad B=h_{0} / \mathrm{WeRe} A^{2}$, and $\alpha=$ $h_{0} / R$. The second term in (2a) is due to a transformation into the frame moving with the velocity of an infinitesimal wave, which is necessary to remove the advective term appearing in the weakly nonlinear expansion. The additional transformation into the frame moving with the velocity of the pulse results in the term $c_{\delta} T$ in the first brackets in (2a).

Equation (1) is the so-called gKS equation. It is the simplest possible prototype that retains the fundamental elements of active-dissipative nonlinear media: the dominant nonlinear term $\left(H H_{X}\right)$, instability $\left(H_{X X}\right)$, stability $\left(H_{X X X X}\right)$, and dispersion $\left(\delta H_{X X X}\right)$, where $\delta=$ $6 / \mathrm{WeRe} A^{1 / 2}$. Let $H_{0}=H_{0}(X)$ be a stationary pulse satisfying the steady version of (1). We assume that $H=$ $\sum_{i=1}^{n} H_{i}+\hat{H}$, where $H_{i}(X, T)=H_{0}\left(X-X_{i}(T)\right)$ is a quasistationary pulse located at $X_{i}(T), i=1, \ldots, n$, and $\hat{H}$ is an overlap function. We consider weak interaction assuming that $L_{i} \equiv X_{i+1}-X_{i}=\log \varepsilon+O(1)$ for $i=$ $1, \ldots, n-1$, where $\varepsilon \ll 1$, and that the velocities of the pulses, $X_{i}^{\prime}, i=1, \ldots, n$, and the overlap function, $\hat{H}$, are of $O(\varepsilon)$. The linearized equation for $\hat{H}$ in the vicinity of the $i$ th pulse takes the form

$$
\hat{H}_{T}-X_{i}^{\prime} H_{i X}=\mathcal{L}_{i} \hat{H}-\alpha_{i}\left(H_{i-1} H_{i}\right)_{X}-\beta_{i}\left(H_{i} H_{i+1}\right)_{X},
$$

where $\alpha_{i}=\beta_{i}=1$ for $1<i<n, \alpha_{1}=\beta_{n}=0$, and $\alpha_{n}=\beta_{1}=1$. $\mathcal{L}_{i}$ s are linear operators defined by $\mathcal{L}_{i} F=$ $c_{\delta} F_{X}-F_{X X}-\delta F_{X X X}-F_{X X X X}-\left(H_{i} F\right)_{X}$. The formal adjoint operators, $\mathcal{L}_{i}^{*} \mathrm{~s}$, with respect to the inner product in $L_{\mathbb{C}}^{2},\langle f, g\rangle=\int_{-\infty}^{+\infty} f \bar{g} d X$, are given by $\mathcal{L}_{i}^{*} F=-c_{\delta} F_{X}-$ $F_{X X}+\delta F_{X X X}-F_{X X X X}+H_{i} F_{X}$. The aim now is to project the dynamics in the vicinity of the $i$ th pulse onto the null space of $\mathcal{L}_{i}$ spanned by the translational mode $H_{i X}$. Our analysis reveals that the null space of $\mathcal{L}_{i}^{*}$ is spanned by a constant and a function $\Psi^{i}$ tending exponentially to different constants as $X \rightarrow \pm \infty$. Therefore, zero is not in the point spectrum of $\mathcal{L}_{i}^{*}$ on an infinite interval. Projections onto the null space of $\mathcal{L}_{i}$ are made rigorously by choosing an appropriate weighted space, namely, $L_{a}^{2}=\left\{f: e^{a X} f \in\right.$ $\left.L_{\mathbb{C}}^{2}\right\}$, where $a$ is a positive sufficiently small number, with the inner product $\langle f, g\rangle_{a}=\left\langle e^{a X} f, e^{a X} g\right\rangle$. Studying the spectrum of $\mathcal{L}_{i}$ in $L_{a}^{2}$ is equivalent to studying the spectrum of the operator defined by $\mathcal{L}_{i}^{a} f=e^{a X} \mathcal{L}_{i}\left(e^{-a X} f\right)$ in $L_{\mathbb{C}}^{2}$ (see Ref. [9]). Zero becomes an isolated eigenvalue of both $\mathcal{L}_{i}^{a}$ and $\mathcal{L}_{i}^{a *}$ of algebraic and geometric multiplicity unity, and projections can now be made in the usual way. Assuming that $\hat{H}$ is in the null spaces of the projections, we arrive at the following system describing the leading-order dynamics of the locations of the pulses:

$$
X_{i}^{\prime}=\alpha_{i} S_{2}\left(X_{i}-X_{i-1}\right)+\beta_{i} S_{1}\left(X_{i+1}-X_{i}\right) ; \quad 1 \leq i \leq n,
$$

where $S_{1,2}(L) \equiv-\int_{-\infty}^{\infty} H_{0}\left(X+\frac{1}{2} L\right) H_{0}\left(X-\frac{1}{2} L\right) \Psi_{X}^{0}(X \pm$ $\left.\frac{1}{2} L\right) d X$. Thus, the position of the $i$ th pulse is governed by its interaction with the oscillatory front of the upstream pulse $(i-1)$, described by $S_{2}$, and with the monotonic tail of the downstream pulse $(i+1)$, described by $S_{1}$. System (4) can be rewritten in terms of the separation distances $L_{i} \mathrm{~s}$ and its fixed points then give the bound states; e.g., for a bound state of two pulses, we must have $S_{1}\left(L_{1}\right)=S_{2}\left(L_{1}\right)$. At the same time, $S_{1,2}$ represent the velocities of the first and of the second pulse relative to $c_{\delta}$, respectively. The graphs of $S_{1}$ and $S_{2}$ are shown in Fig. 3(a) for $\delta=0.4$. Depending on their initial separation distance $L$, pulses either attract $\left(S_{1}>S_{2}\right)$ or repel $\left(S_{1}<S_{2}\right)$ each other. As they get closer or further from each other, their velocity difference decreases until both pulses propagate at the same velocity, forming a bound state. The abscissas of the intersection points hence indicate the separation distances for which
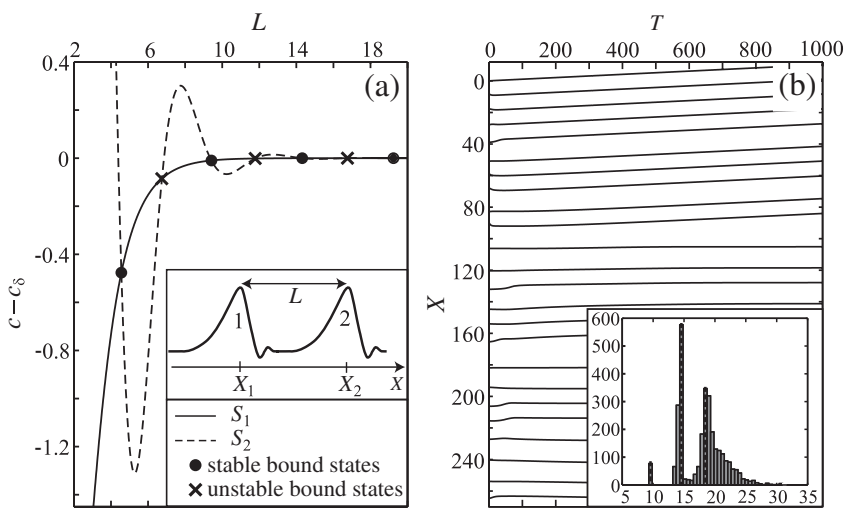

FIG. 3. (a) Dependence of $S_{1}$ and $S_{2}$ on the separation distance between two pulses for $\delta=0.4$. (b) Evolution of pulses of the gKS equation for $\delta=0.4$, in the frame of a solitary pulse obtained by solving (4). Subplot: histogram of the pulse separation distances (the initial distribution was taken to be normal with mean 18 and standard deviation 3 ). 
bound states can be formed. By analyzing the behavior of $S_{1,2}$ as $L \rightarrow \infty$, it can be shown that if $\delta<\delta^{*} \approx 0.85$, there is a countable infinite number of intersections. Otherwise, the number is finite, but for $\delta \gtrsim 1.3$ there are no intersections (we note that for $\delta \gtrsim 1$, numerical experiments with the gKS equation show that we do not have formation of bound states).

We have solved numerically (4) for two pulses and $\delta=$ 0.4 [as in Figs. 2(a) and 2(b)] and compared the results with numerical solutions of (1) when the initial condition is a superposition of two pulses with the same separation distance as that for (4). We found very good agreement between the two. Figure 3(b) shows a typical solution of (4) and the world lines of 24 pulses. We observe both attractions and repulsions as well as formation of twoand three-pulse bound states. Also, the world lines are similar to those obtained in our experiments and shown in Fig. 2(b). The subplot shows a histogram obtained from statistics on 3000 pulse separation distances at $t=1000$. We observe three clear peaks formed at $\simeq 9.5,14$, and 18.5, which are in very good agreement with the stable two-pulse bound state distances shown in Fig. 3(a) (the distance of $\simeq 4$ is disregarded as it corresponds to a two-hump pulse which is never observed in the computations). The inset in Fig. 3(b) reveals a feature that is also present in the experiments [inset of Fig. 2(b)]: the distances between the neighboring prominent peaks in the histograms are roughly the same. The theoretical explanation for this is in Fig. 3(a): $S_{2}$ is oscillatory approaching a sinusoidal function for $L \geqslant 6$ while $S_{1}$ levels off quickly with $L$. Converting the dimensionless distances $9.5,14$, and 18.5 to dimensional ones gives $0.42,0.62$, and $0.82 \mathrm{~cm}$. When compared with the first three peaks occurring in the histogram in Fig. 2(b) at $d_{1} \approx 1.2 \mathrm{~cm}, d_{2} \approx 1.8 \mathrm{~cm}$, and $d_{3} \approx 2.6 \mathrm{~cm}$, we find that the experimental distances are larger than the theoretical ones by a factor of $\simeq 3$. Hence, we have qualitative agreement in that the system appears to select (via both attractions and repulsions) certain separation distances at large times (which are also of the same order with the experiments). However, we do not have quantitative agreement, but this is to be expected as for the theory $R$ was assumed to be large compared to $h_{0}$, which is not the case in experiments, where $\alpha=O(1)$. Violation of this assumption affects significantly the constant $A$ and therefore the rescaling of the distances. Smaller thicknesses are not reachable experimentally: below a critical film thickness of $\simeq 0.5 \mathrm{~mm}$, no flat film can emerge; instead, dripping of the liquid from the faucet is observed. The requirement $\alpha \ll 1$ is approached on a larger radius fiber or with smaller thicknesses on smaller radii; however, under these conditions either $\delta \gtrsim 1$ and we do not observe the formation of bound states as noted earlier or $\delta \lessgtr 0.1$, in which case the behavior is chaotic (we are close to the KS limit). Experimentally, bound states are observed for $0.1 \leqq \delta \leqq$ 1 , when both dispersive and dissipative effects are strong.
This also implies that $\delta$ is indeed the right parameter to measure dispersive effects.

To conclude, we have examined experimentally pulse interaction and formation of bound states on the surface of a film coating a vertical fiber. Theoretical support for these phenomena was provided with a coherent-structures theory for the gKS equation. This equation is not strictly speaking applicable for $\alpha=O(1)$, the regime in which the experiments were conducted, so that a quantitative agreement is not quite achieved. However, it is the simplest possible model for the physical system, containing all its important elements, and thus capable of describing the experiments qualitatively.

We are grateful to C. Ruyer-Quil for insightful comments and suggestions. We acknowledge financial support from EPSRC Grants No. EP/F016492 and No. EP/ F009194, Franco-British Research Partnership Programme, and EU-FP7 ITN Multiflow.

[1] L.E. Ballentine, Quantum Mechanics: A Modern Development (World Scientific, Singapore, 1998); K. Drescher, K. C. Leptos, I. Tuval, T. Ishikawa, T. J. Pedley, and R.E. Goldstein, Phys. Rev. Lett. 102, 168101 (2009); G. A. Voth, B. Bigger, M. R. Buckley, W. Losert, M. P. Brenner, H. A. Stone, and J. P. Gollub, Phys. Rev. Lett. 88, 234301 (2002).

[2] A. L. Frenkel, Europhys. Lett. 18, 583 (1992); S. Kalliadasis and H.-C. Chang, J. Fluid Mech. 261, 135 (1994).

[3] C. Duprat, C. Ruyer-Quil, S. Kalliadasis, and F. GiorgiuttiDauphiné, Phys. Rev. Lett. 98, 244502 (2007); C. RuyerQuil, P. Treveleyan, F. Giorgiutti-Dauphiné, C. Duprat, and S. Kalliadasis, J. Fluid Mech. 603, 431 (2008); C. Duprat, C. Ruyer-Quil, and F. Giorgiutti-Dauphiné, Phys. Fluids 21, 042109 (2009).

[4] T. Kawahara, Phys. Rev. Lett. 51, 381 (1983); C. M. Alfaro, R.D. Benguria, and M.C. Depassier, Physica (Amsterdam) 61D, 1 (1992); C. I. Christov and M. G. Velarde, Physica (Amsterdam) 86D, 323 (1995).

[5] Dissipative Solitons: From Optics to Biology and Medicine, edited by N. Akhmediev and A. Ankiewicz, Lecture Notes in Physics Vol. 751 (Springer, Berlin/ Heidelberg, 2008).

[6] S.-I. Ei, J. Dyn. Differ. Equ. 14, 85 (2002); B. Sandstede, in Handbook of Dynamical Systems II, edited by B. Fiedler (North-Holland, Amsterdam, 2002), p. 983.

[7] C. Elphick, G. R. Ierley, O. Regev, and E. A. Spiegel, Phys. Rev. A 44, 1110 (1991); S.-I. Ei and T. Ohta, Phys. Rev. E 50, 4672 (1994); H.-C. Chang and E. A. Demekhin, Complex Wave Dynamics on Thin Films (Elsevier, Amsterdam, 2002).

[8] Thin Films of Soft Matter, edited by S. Kalliadasis and U. Thiele, CISM Courses and Lectures No. 490 (SpringerWien, New York, 2007).

[9] R. L. Pego and M. I. Weinstein, Phil. Trans. R. Soc. A 340, 47 (1992). 